\title{
Spatial and temporal analysis of neutrophil trans-epithelial migration in response to RSV infection
}

Authors: Elisabeth Robinson ${ }^{1}$, Jenny Amanda Herbert ${ }^{1,2}$, Machaela Palor ${ }^{1}$, Luo Ren ${ }^{1,3}$, Isobel Larken ${ }^{1}$, Alisha Patel ${ }^{1}$, Dale Moulding ${ }^{1}$, Mario Cortina-Borja ${ }^{1}$, Rosalind Louise Smyth $^{1 \dagger}$, Claire Mary Smith ${ }^{1 \dagger^{*}}$.

Affiliations:

${ }^{1}$ UCL Great Ormond Street Institute of Child Health, London, UK.

${ }^{2}$ School of Medical Sciences, Faculty of Biology, Medicine \& Health, University of

Manchester, UK.

${ }^{3}$ Department of Respiratory Medicine, Children's Hospital of Chongqing Medical University, Chongqing, 400014, China

† joint senior author

* corresponding author:

Dr Claire Mary Smith,

UCL Great Ormond Street Institute of Child Health,

30 Guilford St, London WC1N 1EH, c.m.smith@ucl.ac.uk

\section{Keywords:}

Manuscript word count 


\section{Abstract}

In the airways, recruitment and activation of neutrophils occurs early following respiratory virus (RSV) infection and is associated with the development of severe disease. We investigated whether activated neutrophils selectively migrate across virus infected airway epithelial cells, or whether trans-epithelial migration is sufficient and necessary for neutrophil activation. We profiled the movement and adherence of fluorescently labelled human neutrophils during migration across primary human airway epithelial cells (AECs) infected with RSV in vitro. In RSV infected AECs neutrophil adherence, with clustering occurs after 15-18 minutes. Using flow cytometry, we found that, when migration occurred, expression of CD11b, CD62L, CD64, NE and MPO were increased in all compartments of our system and RSV infection further increased CD11b and NE expression. We found evidence suggesting that migrated neutrophils can migrate in reverse to the basolateral membrane. Our study provides novel insights into how airway activated neutrophils mediate systemic disease in respiratory virus infection.

\section{Abstract word count (approx 150)}




\section{Inserts with a $3 \mu \mathrm{m}$ pore size permit trans-epithelial migration}

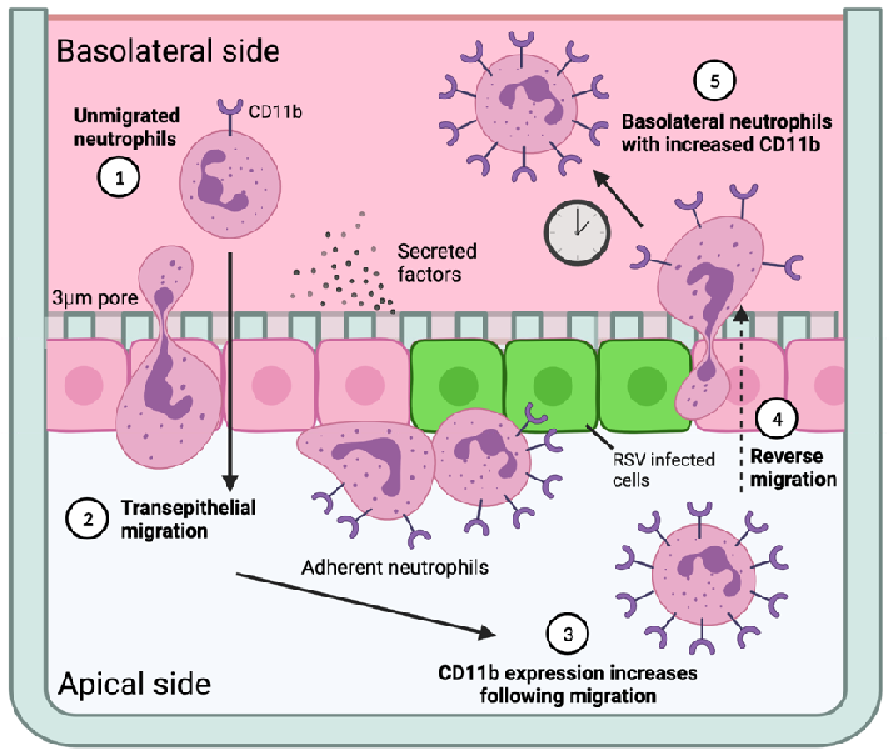

\section{$0.4 \mu \mathrm{m}$ pores prevent trans-epithelial migration}

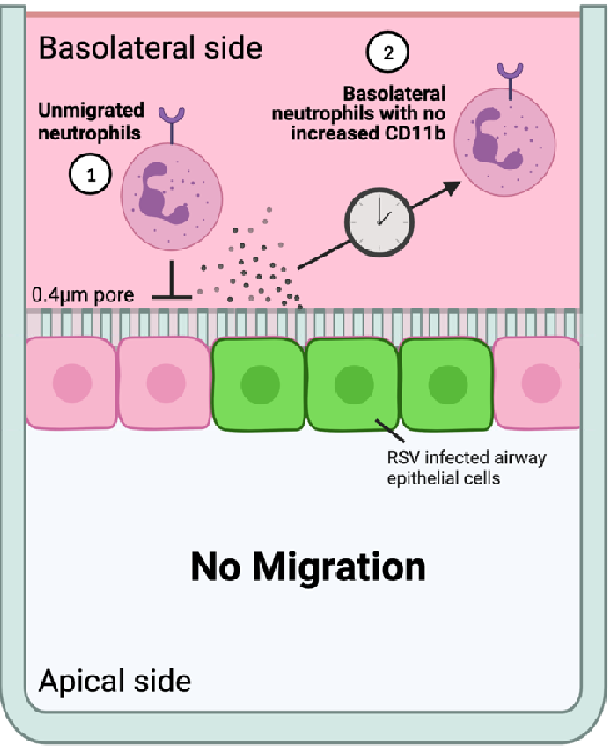

Graphical Abstract Legend - Air-liquid interface (ALI) models for studying neutrophil trans-epithelial migration in response to RSV infection. Left panel shows primary airway epithelial cells cultured at ALI on membrane inserts with $3 \mu \mathrm{m}$ pore size that permits neutrophils to migrate through. 1 ) unmigrated neutrophils expressing baseline levels of CD11b, 2) neutrophils migrate across infected $A E C s$ and some remain adherent to the infected AECs, 3) neutrophils are shown to increase expression of CD11b and other activation associated markers, 4) some 'activated' neutrophils undergo reverse migration as 5) neutrophils with the increased expression of CD11b are detected on the basolateral side of the insert. Right panel shows primary airway epithelial cells cultured at ALI on membrane inserts with $0.4 \mu \mathrm{m}$ pore size that prevents neutrophils migration. Here, after $1 \mathrm{~h}$ incubation with RSV infected AECs, neutrophils on the basolateral side 1) and 2) were shown to express the same level of CD11b markers, indicating that neutrophil migration is key process for increasing expression of these activation markers. Drawing created using BioRender.com. 


\section{Introduction}

Respiratory Syncytial Virus (RSV) is a seasonal respiratory virus, reported to infect almost all children before the age of $2^{1}$. Following infection, most children develop an illness which is confined to upper respiratory tract symptoms, however $1-3 \%$ of infants infected will develop a severe illness requiring hospitalisation ${ }^{2}$. There is no licensed vaccine for RSV and treatment is currently limited to supportive care. In resource-limited countries, RSV is a major cause of infant death ${ }^{3-5}$.

Although risk factors have been identified, it is still not clear why, RSV infected infants with no apparent risk factors, require hospitalisation and respiratory support ${ }^{6}$. One suggestion is that neutrophils, which form around $80 \%$ of all cells recovered from the airways of infants with severe RSV bronchiolitis by bronchoalveolar lavage ${ }^{7}$, and their associated cytokines contribute to disease severity ${ }^{8-10}$. Neutrophil mediated factors such as CXCL8 (IL-8), CXCL10 (IP-10) and neutrophil elastase are present in substantial amounts in airway secretions of children with severe RSV bronchiolitis. ${ }^{11-13}$.

Neutrophils are the predominant immune cell type in the systemic circulation and are the first cellular component of the innate immune system to migrate from the bloodstream through the airway epithelium during infection ${ }^{14}$. This recruitment and movement through the airway epithelium are facilitated by receptors on neutrophils including integrins (i.e. CD11b) and selectins (such as CD62L) that bind to molecules on airway epithelial cells such as ICAM$1^{15-17}$. Once at the site of infection, neutrophils are thought to employ largely non-specific mechanisms of pathogen destruction including phagocytosis, neutrophil extracellular trap formation and release of toxic granule products such as neutrophil elastase and myeloperoxidase ${ }^{18,19}$. Neutrophils also interact with other immune complexes using Fc receptors such as CD64; upregulation of CD64 has been evaluated as a biomarker for neonatal sepsis in infants ${ }^{20}$. Although RSV is not known establish infection outside the respiratory tract or cause a viraemia, our group has shown, in clinical studies, that a high proportion of neutrophils from the systemic circulation of infants with RSV bronchiolitis contain RSV mRNA ${ }^{11}$. This raises the possibility that neutrophils, recruited to the airway, during RSV infection, may be able to migrate, in the reverse direction, to the systemic circulation. 
Our previous work has shown that neutrophils migrate across primary differentiated AECs and their adherence to RSV infected AECs is associated with increased epithelial damage and a reduced viral load ${ }^{21,22}$. Interestingly, we also observed that neutrophils adherent to RSV infected AECs in a clustering pattern, which was not seen in uninfected AECs.

Previous reports, using intravital microscopy, have shown evidence of co-ordination between neutrophils during chemotaxis and migration. These patterns have been referred to as 'neutrophil swarming' due to a resemblance with the swarming behaviour of insects ${ }^{23,24}$.

This study further examines the effect of RSV infection on the behaviour and function of neutrophils as they move through the epithelial cell layer. We studied this migration using an undifferentiated AEC model, ${ }^{22}$ in order facilitate the higher-quality z-stack microscopy needed to track neutrophil migration. (Figure 1). We evaluate the kinetics of neutrophil motility during trans-epithelial migration and 'cluster formation' between RSV infected and uninfected AECs.

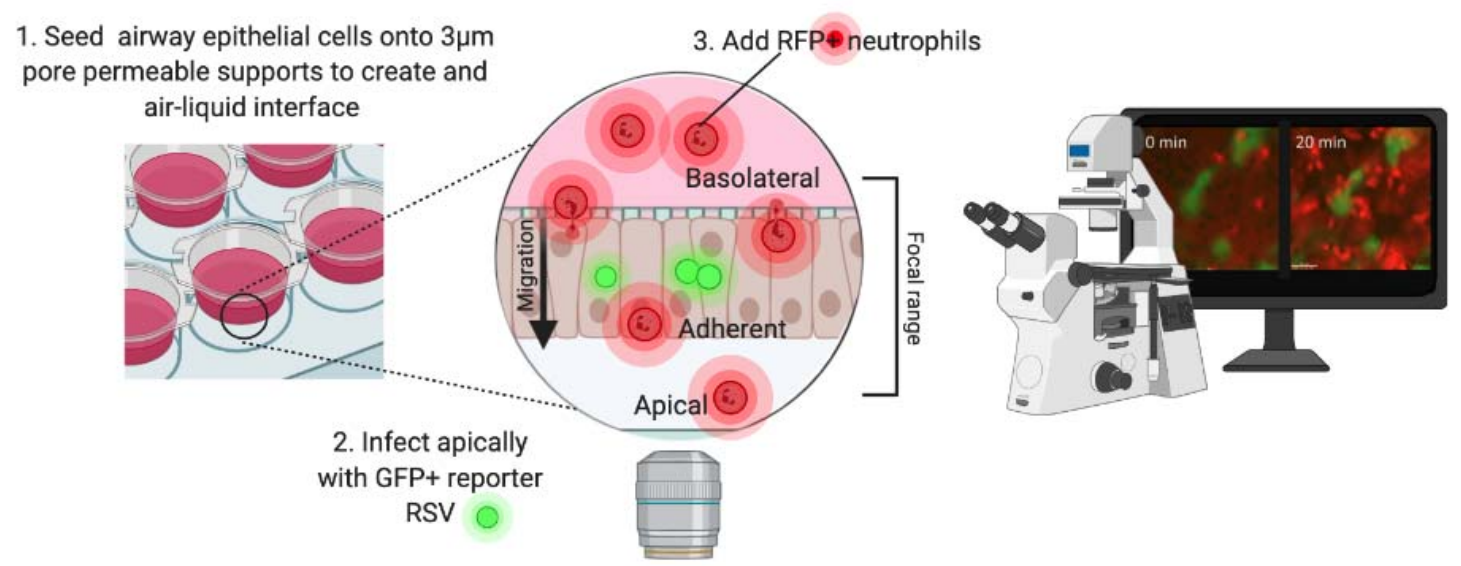

Figure 1 - Schematic of method and model used to study neutrophil trans-epithelial migration in response to RSV infection. Human primary airway epithelial cells (AECs) were cultured on the underside of a $3 \mu \mathrm{m}$ pore size, transparent PET culture membrane insert. AECs were matured at an air-liquid interface (ALI) for 7 days before infection with GFP tagged RSV for 24 or 72 hours. At this time, neutrophils, stained with a viability stain (RFP), were added to the basolateral side of $A E C$ cultures and a $50 \mu \mathrm{m}$ Z-stack image of the focal area indicated was captured for up to $1 \mathrm{hr}$. 


\section{Results}

Neutrophil migration increases release of neutrophil proteases and damage to airway epithelial cells.

Using a primary human airway epithelial cell model (Figure 1) we investigated the movement of neutrophils as they migrate across RSV infected epithelial cells that were grown at air-liquid interface for 7 days. As controls, we compared neutrophil migration across either 1) mock infected AECs treated as the RSV infected AECs, 2) mock infected AECs exposed to potent neutrophil chemoattractant (fMLP), or 3) mock infected AECs exposed to RSV infected AEC supernatant (referred to as RSV Sup). These controls allowed us to differentiate whether our observations were due to the process of migration per se or to inflammatory mediators present in the RSV infected airway supernatant.

Firstly, as we have done before in differentiated cultures ${ }^{22}$, we characterised whether neutrophil migration increases epithelial damage in this undifferentiated model. We found that $1 \mathrm{~h}$ after neutrophil trans-epithelial migration across epithelium infected with RSV for $24 \mathrm{~h}$, we detected larger gaps with mean \pm SEM of $70.8 \pm 4.6 \%$ area in the RSV infected epithelial layer compared to the mock-infected $(61.6 \pm 6.0 \%$ area) $(p<0.0001)$ (representative images shown in Figure 2A). We also detected a loss of RSV infected cells, as is observed in Video Stills A. Here as neutrophil transepithelial migration progresses, an AEC expressing GFP RSV indicated by a yellow circle, disappears from the image.

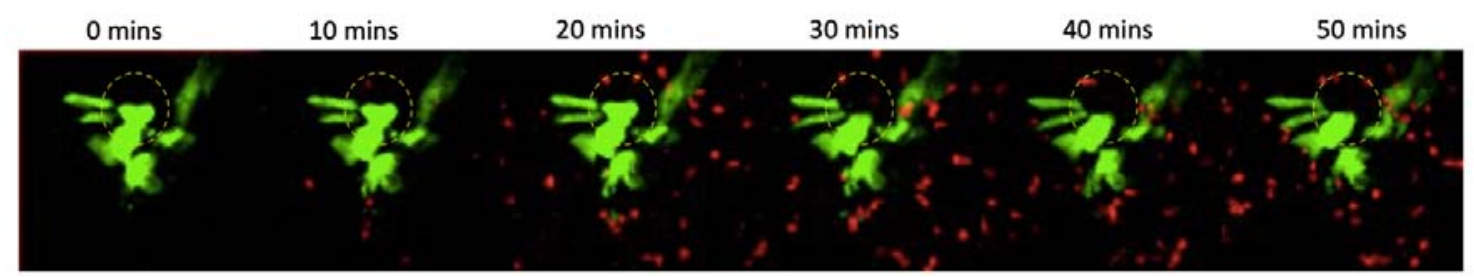

Video Stills A- Elimination of RSV infected AEC during neutrophil migration through

RSV infected AECs. Representative stills of a time lapse stack detailing neutrophil migration through RSV infected AECs. Green indicates RSV infected cells expressing GFP and red indicates neutrophils stained with calcein red-orange. Yellow dashed circle shows position of RSV-infected epithelial cell.

Neutrophils release several toxic products, including myeloperoxidase (MPO) and neutrophil elastase (NE). We measured the concentration of these products in the compartments of our model after neutrophil migration. We found that the concentration of NE, in airway surface media of AEC cultures, was $>3$ fold greater than the basolateral media following neutrophil 
migration across RSV infected epithelium for $4 \mathrm{~h}$ at $72 \mathrm{~h}$ post infection (Figure 2B) with a mean $\pm S E M$ of $2.0 \pm 0.6 \mathrm{mU} / \mathrm{ml}$, compared to $0.6 \pm 0.1 \mathrm{mU} / \mathrm{ml}$ in the mock-infected cultures $(p=0.039)$ (Figure 2B). We did not find a significant difference in MPO in airway surface media from RSV infected cultures following neutrophil migration for $4 \mathrm{~h}$ compared to the mock-infected cultures.

As we previously found that increased neutrophil adherence was associated with increased epithelial damage to RSV infected ciliated cultures ${ }^{22}$, we counted the number of neutrophils that migrate across and adhere to AECs by labelling neutrophils with a fluorescent viability stain and imaging adherent neutrophils using fluorescence microscopy (Figure 2C). Total counts of viable neutrophils recovered basolaterally, apically and adherent to the AECs is shown for reference (Figure 2D). Because we detected loss of AECs and a drop in epithelial integrity after 4 hours here and in our previous study ${ }^{22}$, which may distort measurements of neutrophil migration due to passive leakage of cells and or secreted factors, all subsequent experiments report findings of neutrophil adherence during the first hour of migration.

Here we found that the numbers of neutrophils adherent to AEC cultures infected with RSV for $24 \mathrm{~h}\left(791.1 \pm 106.8 \mathrm{cells} / \mathrm{cm}^{2}\right)$ were significantly $(p=0.0135)$ greater than the respective mock infected AEC cultures (449.8 $\pm 81.82 \mathrm{cells} / \mathrm{cm}^{2}$ ) (Figure 2E). Similarly, AEC cultures infected with RSV for 72 hours showed significantly $(p=0.0152)$ greater numbers of adherent neutrophils $\left(711.1 \pm 74.3 \mathrm{cells} / \mathrm{cm}^{2}\right)$ compared to the respective mock control $\left(486.6 \pm 61.5 \mathrm{cells} / \mathrm{cm}^{2}\right)$ (Figure $\left.2 \mathrm{E}\right)$. However, significantly $(p=0.0056)$ fewer neutrophils remained adherent when migrating across mock infected AECs exposed to RSV infected AEC supernatant $\left(462.7 \pm 43.3\right.$ cells $/ \mathrm{cm}^{2}$ ), in comparison to the 72 -hour RSV infected $(711.1$ $\pm 74.3 \mathrm{cells} / \mathrm{cm}^{2}$ ) (Figure 2E), suggesting that the presence of RSV infected epithelial cells, rather than soluble, secreted factors alone, is important in RSV induced neutrophil adherence.

In addition to this, we measured the numbers of neutrophils that migrated across and dissociated from the epithelium, as an indication of neutrophils that may be present in the airway lumen. Interestingly, we found that there was no significant difference in the number of apical neutrophils that migrated across cultures infected with RSV for $24 \mathrm{~h}$ with an average (mean \pm SEM) of 22,876 neutrophils/well \pm 12713 , or $4.4 \%$ of the total neutrophils added, compared to the relative mock infected AEC cultures $(19,184$ neutrophils/well $\pm 10,806)$ or $3.8 \%$ of total (Figure $2 F)$. However, we recovered significantly $(p=0.006)$ more neutrophils from mock infected AEC cultures exposed to supernatant collected from AEC cultures 
bioRxiv preprint doi: https://doi.org/10.1101/2021.10.04.463016; this version posted October 5, 2021. The copyright holder for this preprint (which was not certified by peer review) is the author/funder, who has granted bioRxiv a license to display the preprint in perpetuity. It is made available under aCC-BY-NC-ND 4.0 International license.

infected with RSV for 24 hours (RSV Sup), with an average 70,016 cells/well $\pm 21,115$ compared to the mock with 19,184 cells/well $\pm 10,806$ and 22,876 cells/well $\pm 12,713$ in comparison to RSV ( $p=0.0058$ ) (Figure 2F). When migrated through AECs infected with RSV for 72 hours, these differences were similar; $(p>0.05)$ the number of neutrophils recovered from the RSV infected AEC cultures $(40,415$ cells/well $\pm 15,143)$ compared to the mock $(59,900$ cells/well $\pm 18,885)$ after 1 hour incubation (Figure 2F). Although more neutrophils were recovered from the apical side of RSV infected AECs, a greater number were recovered apically from mock infected AECs exposed to fMLP $(170,000$ cells/well \pm 25,212), suggesting that, while RSV infected AEC supernatant has a chemotactic effect and increases migration, it was not as greater effect as with the positive control $\mathrm{FLP}$.

Figure 2 (Overleaf) RSV increases the numbers of neutrophils that adhere to RSV infected epithelial cultures, forming clusters on the AEC surface. (A) - Representative image showing neutrophil (red cells) adherence to epithelial cells (DAPI staining - blue) following migration for $1 \mathrm{~h}$ at $24 \mathrm{~h}$ or $72 \mathrm{~h}$ post RSV or mock infection. fMLP $1 \mathrm{ng} / \mathrm{ml}$ placed apical to uninfected AECs was used a positive control for neutrophil chemotaxis. Scale bar indicates $50 \mu \mathrm{m}(\mathrm{B})-$ Soluble granular factors myeloperoxidase (MPO), neutrophil elastase (NE) and matrix-metalloproteinase-9 (MMP-9) in the apical supernatant (designated AS in figure) and basolateral supernatant (BS) were quantified using ELISA. fMLP and mock infected AECs with supernatant collected from RSV infected AECs placed apically (RSV Sup). (C) Representative 2 channel maximum intensity projection of a Z-stack image

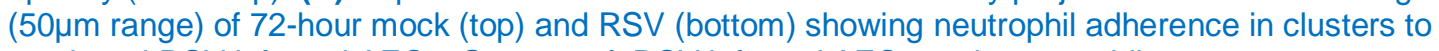
mock and RSV infected AECs. Green = gfpRSV infected AECs, red=neutrophils. Scale bar indicates $100 \mu \mathrm{m}$ (D) - Graph showing representative counts of neutrophils isolated either basolateral to, apical to or adherent to AECs. Absolute counts performed using a flow cytometer selecting for neutrophils as positive for CD11b-APC antibody staining. (E) - Numbers of neutrophils adherent to AECs after $1 \mathrm{hr}$ transepithelial migration across 24 or $72 \mathrm{hr}$ RSV infected AECs. Adherent neutrophils and epithelial cells were counted using Image $\mathrm{J}$ counting tool, the average number of neutrophils from all images is shown. (F)- Numbers of neutrophils migrating and dissociating apically from AECs after $1 \mathrm{hr}$ transepithelial migration across 24 or 72 hr RSV infected AECs. Neutrophil concentrations were quantified in the apical surface media using a plate reader and read against a standard curve. 


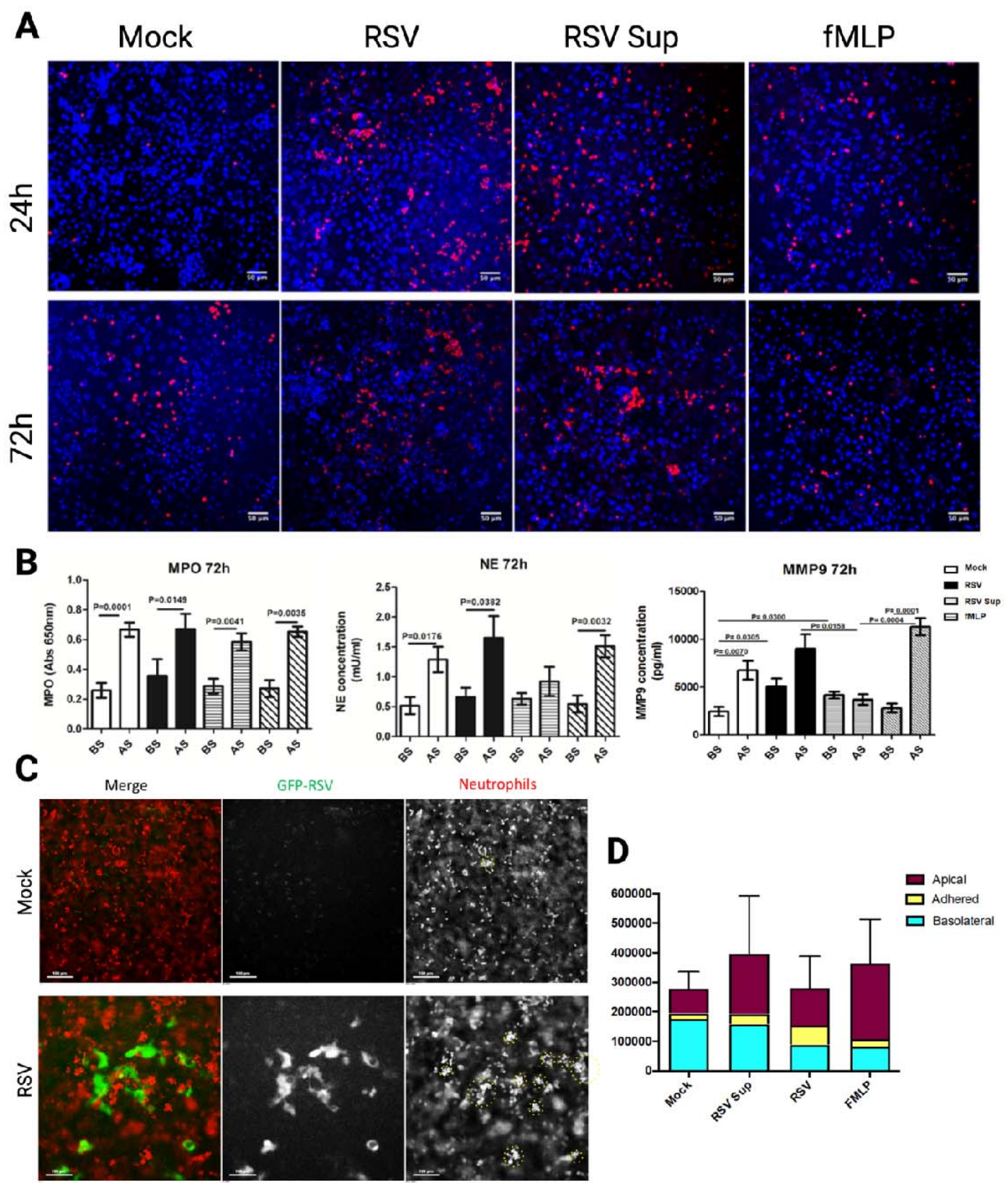

$\mathbf{E}$

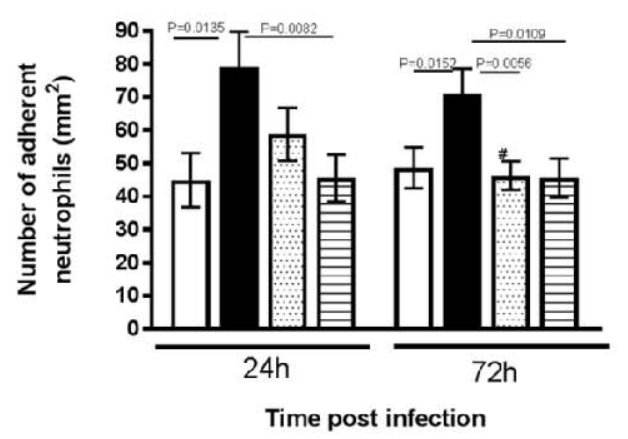

$\mathbf{F}$

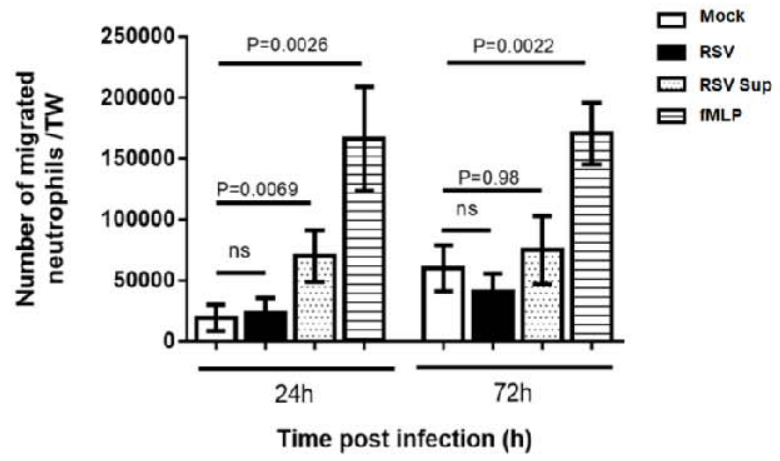




\section{Neutrophils adherent to the RSV infected AECs are more likely to be}

\section{'clustered' compared to neutrophils adherent to mock infected AECs}

After quantifying neutrophil trans-epithelial migration across RSV infected AECs, we sought to measure the distribution of neutrophil on the surface of RSV-infected AECs. We

previously showed that neutrophils adherent to RSV infected epithelium appeared to form large clusters ${ }^{22}$. To investigate this further, we used vector analysis (or XYZ coordinates) to determine the 3D location of each neutrophil and measured the distance from each to their nearest neighbours (Supplementary Figure 1A). To account for spatial differences that may be due to the greater numbers of adherent neutrophils in RSV-infected, compared with mock-treated cultures (Figure 2B), we calculated the expected nearest neighbour median distance, assuming an even distribution of the average number of adherent neutrophils. The expected nearest neighbour median distance was shorter in RSV infected AEC cultures $(140 \mu \mathrm{m} \pm 24.3)$ in comparison to neutrophils adherent to the mock infected epithelium

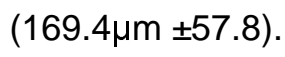

We found that the observed nearest neighbour median distance between neutrophils adherent to mock infected AECs $(119.4 \mu \mathrm{m} \pm 51.6)$ and the expected distance $(169.4 \mu \mathrm{m}$ $\pm 57.8)$, this difference was significant ( $p=0.004)$ (Figure 3A). Similarly, comparing the observed distances between neutrophils adherent to RSV infected AECs $(61.32 \mu \mathrm{m} \pm 30.9)$ after $1 \mathrm{~h}$ migration was significantly $(\mathrm{p}<0.0001)$ shorter than the expected distance $(140 \mu \mathrm{m} \pm$ 24.3) (Figure 3B). This indicates that the distribution of these adherent neutrophils on the epithelial surface is neither random nor uniform.

\section{Temporal analysis of neutrophil trans-epithelial migration reveals neutrophil swarming.}

After quantifying neutrophil distribution on RSV infected AECs after 1h, we sought to observe the early interaction and possible formation of neutrophil clusters over time. In order to directly compare neutrophil movement across RSV and mock infected AECs, we used a using a Zeiss LSM 710 confocal microscope, which allowed us to concurrently capture 2 test

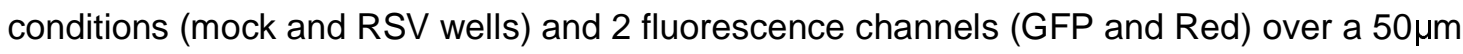
z-range every 2 minutes. A video of neutrophil migration across RSV and mock infected AECs are shown in VIDEOS 1 and 2 respectively. 

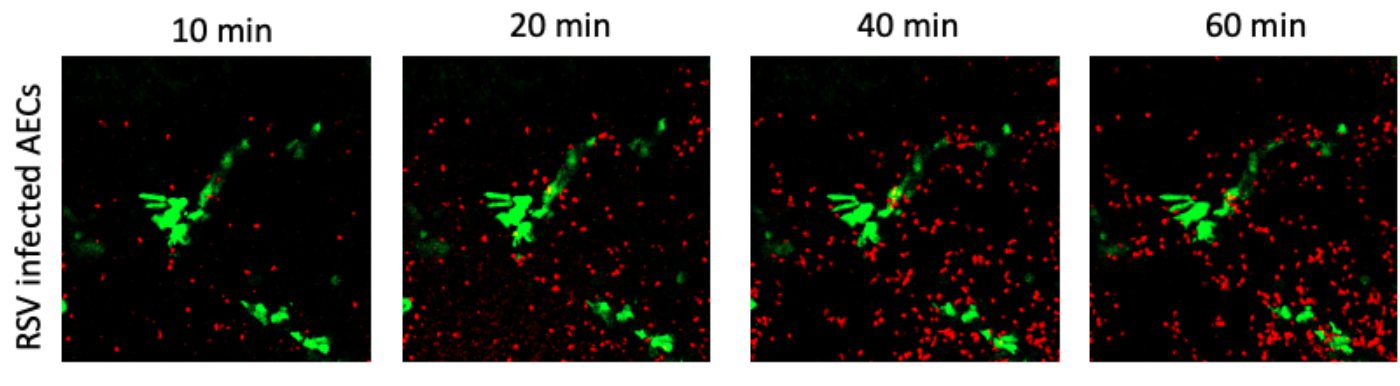

Video 1 Time-lapse multichannel video of neutrophils migrating through RSV infected AECs. Neutrophils added at $t=0$ and a multichannel stack image taken every 2 mins for 1 hour. Green $=$ GFP RSV infected cells. Red = calcein red-orange stained neutrophils

$10 \min$

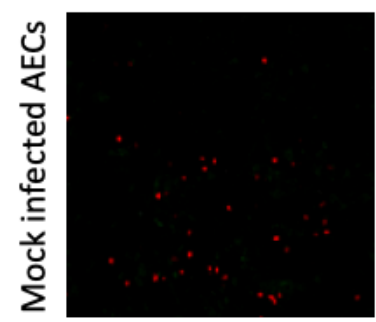

$20 \min$

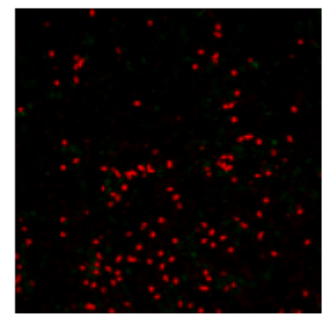

$40 \mathrm{~min}$

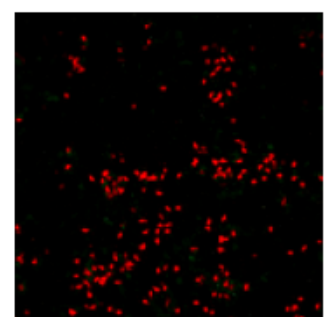

$60 \mathrm{~min}$

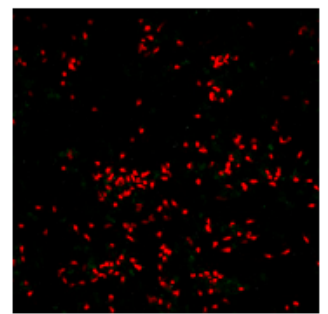

Video 2 Time-lapse multichannel video of neutrophils migrating through mock infected AECs. Neutrophils added at $t=0$ and a multichannel stack image taken every 2 mins for 1 hour. Green $=$ GFP RSV infected cells. Red $=$ calcein red orange-stained neutrophils

Using vector analysis we found that, after 20 minutes of migration, the observed median ( \pm IQR) difference between neutrophils and their nearest neighbours $(102.9 \mu \mathrm{m} \pm 55.8)$ was significantly shorter $(p=0.0008)$ than the distance expected by chance alone $(140 \mu \mathrm{m} \pm$ 24.3) (Figure 3C). This suggests that neutrophil clustering begins to occur around 20 mins after the addition of neutrophils to the basolateral side of AECs in our model. Between 10-20 minutes we detected a more marked decline in nearest neighbour distance to $42.9 \mu \mathrm{m} \pm 55.8$. After this time phase, and between 20-60 minutes, the mean distance to nearest neutrophil stays reasonably constant around $50 \mu \mathrm{m}$ (Figure $\mathbf{3 C}$ ).

To attempt to improve the time resolution of when, during trans-epithelial migration, neutrophils begin to cluster, we imaged neutrophil movement across RSV infected AECs using a fast time-lapse capture (every 45 seconds) on a Perkin Elmer UltraView spinning disk (CSU22) confocal microscope, a video of which is shown in Video 3. Here, we observed the rapid clustering of neutrophils which began as early as 4 minutes after the 
addition of neutrophils. This neutrophil movement bears resemblance to previous in vivo work which described this observation as neutrophil swarming ${ }^{24}$.
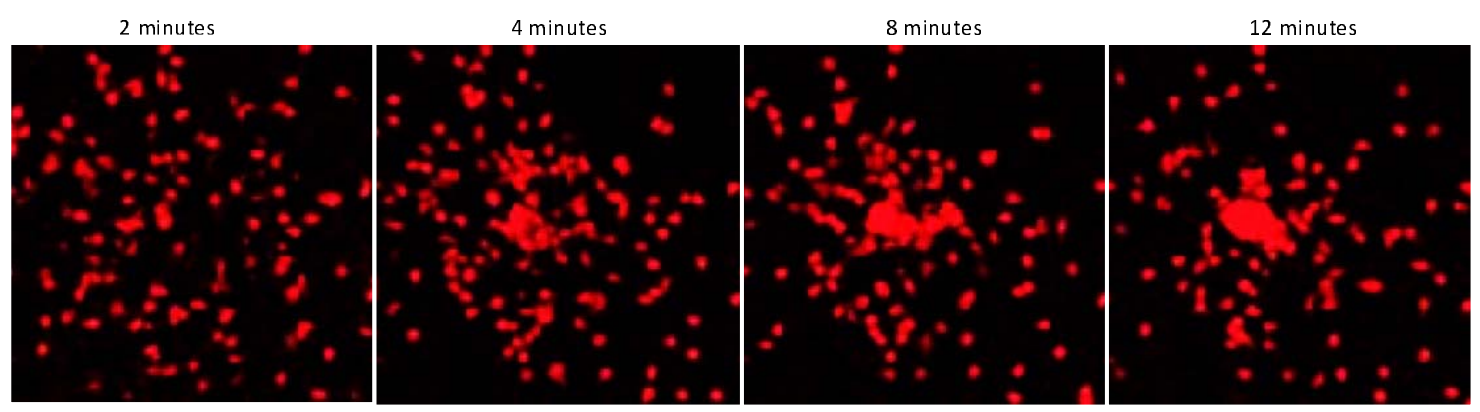

Video 3 Fast time-lapse of neutrophil cluster formation during transepithelial migration across RSV infected AECs. Fast time lapse stills of neutrophil (red stained) cluster formation over time for 1 hour, red $=$ neutrophils stained with calcein red orange. Image captured using a Perkin Elmer Spinning disk

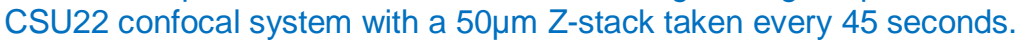

We then measured the track duration and distance travelled of individual neutrophils $(n=200$ per condition) during movement through mock and RSV infected AECs, to determine whether differences in neutrophil adherence could be associated with the speed of neutrophil migration. We found that, in the first 15 minutes of exposure, there was no significant difference in speed of neutrophils migrating across RSV and mock infected AECs (Figure 3D). However, over the course of the first hour, neutrophils' average (mean $\pm S E M$ ) speed when moving through RSV infected AECs was significantly $(p<0.0001)$ slower $(2.28 \mu \mathrm{m} / \mathrm{sec} \pm 0.008)$ than the average speed of neutrophils moving through the mock infected AECs $(4.18 \mu \mathrm{m} / \mathrm{sec} \pm 0.14)$ (Figure 3D). Interestingly, we performed some preliminary analysis of the Z-tracks of neutrophils migrating across RSV infected AECs (Supplementary Figure S4). This showed that neutrophils can move bidirectionally across the AECs, migrating to the apical side of RSV infected airway epithelium and returning to the basolateral compartment, as soon as 15 minutes after migration. The exact proportions of reverse migrating neutrophils and comparisons to mock-infected AEC model remains to be determined.

Observing mean square displacement of neutrophils moving across mock and RSV infected AECs over time, showed for the first 20 minutes neutrophils moving across mock infected AECs appeared to displace further than those moving across RSV infected AECs but this difference became less pronounced after 20 minutes (Figure 3E). We found no significant difference in linearity, or 'track straightness' between neutrophils observed migrating through RSV infected epithelium or the mock (Figure 3F). However, neutrophils moving through 
mock infected AECs showed significantly $(p<0.0001)$ greater total displacement $(137.19 \mu \mathrm{m}$ \pm 4.51 ) compared to those moving through RSV infected AECs $(107.05 \mu \mathrm{m} \pm 4.86)$ (Figure $3 \mathbf{G ) .}$

To disassociate the effect of the AECs from secreted factors in the airway surface media on neutrophil migration, we plotted the $X Y$ movement of naive neutrophils towards apical supernatants using specialised 2D chemotaxis chambers (Supplementary Figure 1B). Here, we found that neutrophils exposed to supernatants collected from AEC cultures infected with RSV for 72 hours $(0.205 \mu \mathrm{m} / \mathrm{sec} \pm 0.001)$ moved faster $(p<0.05)$ than neutrophils exposed to supernatant collected from 72-hour mock treated AECs $(0.110 \mu \mathrm{m} / \mathrm{sec} \pm 0.001)$ (Supplementary Figure 1C). At their fastest recorded speed, neutrophils exposed to supernatants collected from AECs infected with RSV for 72 hours $(0.568 \mu \mathrm{m} / \mathrm{sec} \pm 0.049)$ were $1.5 \times$ faster $(p=0.018)$ than those migrating toward supernatant collected from 72 -hour mock treated AECs $(0.377 \mu \mathrm{m} / \mathrm{sec} \pm 0.014)$. There was no significant difference between the neutrophils exposed to media only, or exposed to supernatants from AEC cultures infected with RSV for 24 hours or mock treated for the same period.

Measuring cumulative displacement (i.e., total distance travelled), we found that neutrophils exposed to supernatants collected from AECs infected with RSV for 72 hours ( $368 \mu \mathrm{m} \pm$ $49.9)$ or 24 hours moved significantly further $(p=0.016)(349 \mu \mathrm{m} \pm 49.9)$ than those exposed to media alone $(182.7 \mu \mathrm{m} \pm 8.96)$ (Supplementary Figure 1D). 
A

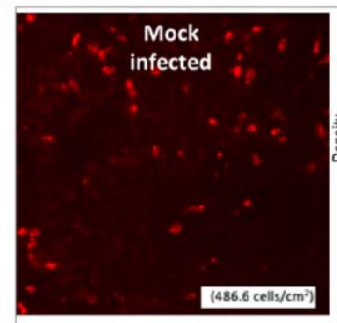

C

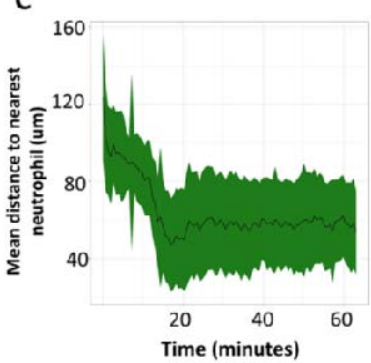

$\mathbf{F}$

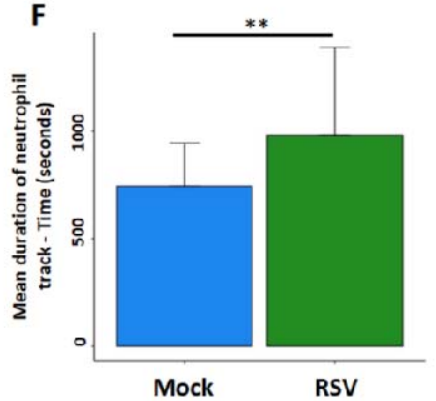

B

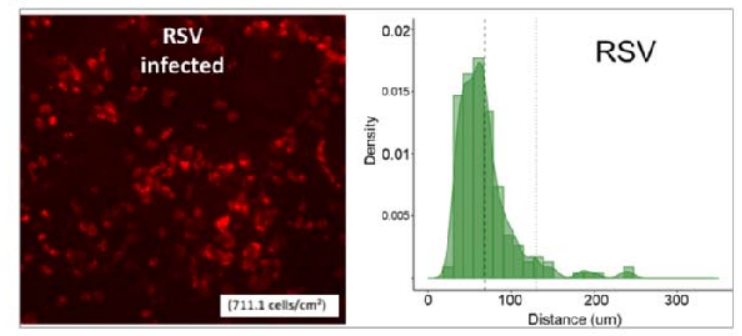

$\mathrm{E}$

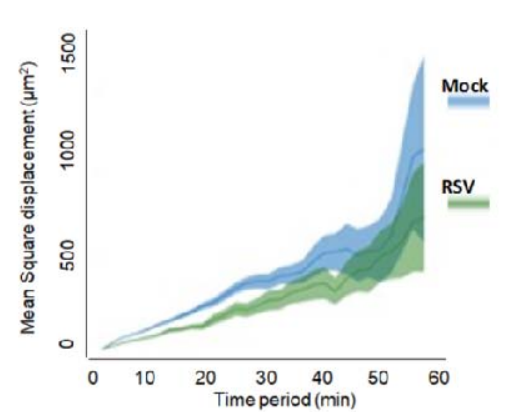

Figure 3 Neutrophil movement during migration across RSV infected AECs displays a different pattern to uninfected AECs. (A \& B)- Distribution analysis of adherent neutrophils to the RSV (right, green) and Mock (left, blue) infected AECs after 1-hour trans-epithelial migration. AECs were fixed after 1 hour neutrophil migration; neutrophil position was processed using ImageJ and coordinates analysed using $R$ version 4.0.3. (Right) Histogram shows the distribution of distance to nearest neighbouring neutrophil calculated from neutrophils adherent to the RSV or mock infected AECs after 1 hour's migration. Thin dashed lines show median distance to nearest neighbouring neutrophil. Thick dashed lines show median distance to nearest neighbouring neutrophil if distribution was assumed to be even based on numbers of neutrophils counted adherent. Median distances were compared using a Wilcoxon-Mann- Whitney $U$ test. (C) Mean distance of each neutrophil to its nearest neighbour over time during migration through RSV infected AECs, calculated as above from fast time-lapse in Video 4 (D)- Speed of neutrophil during movement across mock and RSV infected AECS, split into 15minute segments calculated using motilityLab. All further track metrics calculated using motilityLab. (E) - Mean squared displacement of neutrophils at each time point during movement across mock and RSV infected AECS. Statistical analysis between groups were performed using a Wilcoxon-MannWhitney $U$ test, where significance was found this is indicated on the chart. ${ }^{* * *} p<0.0001$. (F) Mean track duration of individual neutrophils during movement through mock and RSV infected AECs (G) Mean linearity of neutrophil movement during migration through mock and RSV infected AECs 


\section{Neutrophils upregulate surface markers following migration across AECs}

So far, this study has shown that neutrophils are capable of trans-epithelial migration across undifferentiated airway epithelial cells and either become adherent to AECs or migrate and dissociate into the apical space; whilst others 'remain' on the basolateral side (see Figure 1). To determine whether the ability of a neutrophil to migrate and adhere to epithelial cells is associated with specific neutrophil surface marker expression, we recovered neutrophils from basolateral, adherent, and apical compartments after 1 hour and analysed cellular markers associated with neutrophil activation and migration (CD11b, CD64, CD62L, NE, MPO) using flow cytometry. We chose the 72-hour timepoint following AEC infection, and $1 \mathrm{~h}$ timepoint after neutrophil migration for these experiments as these were the conditions that resulted in the greatest number of adherent neutrophils (Figure 2E) to allow effective comparisons.

Firstly, as a control, we determined whether naïve neutrophils were stimulated by apical supernatants recovered from mock or RSV infected AECs (Figure 4). We found a significantly greater expression of CD11b on neutrophils incubated with RSV infected AEC supernatant $(4,078.3 \pm 109.8)$ than neutrophils incubated with media alone $(1,583.7 \pm 34.3)$ $(p=0.0002)$. We also found a significantly greater expression of CD11b on neutrophils incubated with $72 \mathrm{hr}$ RSV infected AEC supernatant (4,078.3 \pm 109.8$)$ in comparison to neutrophils incubated with $72 \mathrm{hr}$ mock infected AEC supernatant $(2,751 \pm 37.5)(p=0.001)$

(Figure 4A). There was no significant difference in CD11b expression between neutrophils incubated with mock infected AEC supernatants and neutrophils incubated with media alone. There was also no significant difference in CD62L, CD64, NE or MPO expression on neutrophils incubated with supernatants from mock and RSV infected AECs (Figure 4BCD\&E).

Using our AEC model, we found that the expression of CD11b, CD64 and CD62L were significantly $(p<0.05)$ higher in neutrophils recovered from basolateral, adherent, apical compartments of both mock and RSV infected AEC models compared to neutrophils exposed to media alone (non-AEC control) (Figure 4ABC) (comparison not directly shown on graph). RSV infection led to 1.5 -fold greater $(p=0.021)$ CD11b expression on basolateral $(54,389 \pm 3,863)$ and apical $(104,145 \pm 6,631)$ neutrophils compared to neutrophils recovered from the respective compartments of mock infected cultures $(36,708 \pm 3,563 \&$ $61,466 \pm 4,876$ ) (see Figure 4A). There was no significant difference in CD64, CD62L, NE, MPO expression on neutrophils recovered from RSV compared to mock infected AECs (Figure 4BCD\&E). The highest expression levels for CD11b were recorded on apical neutrophils recovered from RSV infected AECs $(104,145 \pm 6,631)$, which was more than 
two-fold higher $(p<0.05)$ than both basolateral neutrophils $(54,389 \pm 3,863)$ and adherent neutrophils $(53,561 \pm 3932)$ in the same model and $>10,000$-fold higher than neutrophils in media alone (no-AEC) $(1,583.7 \pm 34.3)$ (Figure 4A).

Interestingly, we found that neutrophils recovered from the basolateral compartment of both mock $(36,708 \pm 3,563)$ and RSV infected AECs $(54,389 \pm 3,863)$ and had 24 and 30-fold greater $(p<0.05)$ CD11b expression, respectively, compared to neutrophils recovered media alone (no-AECs) $(1,583.7 \pm 34.3)$ (see Figure 4A). To determine whether this was due to factors secreted into the basolateral compartment by the AECs, and therefore independent of migration, additional experiments where AECs were grown on membranes with a $0.4 \mu \mathrm{m}$ pore size. This smaller pore size does not permit neutrophils to move across the membrane to the apical side of AECs (Supplementary Figure S2) but will allow for passive diffusion of secreted factors. Using this system, we found that the expression of CD11b, CD64, CD62L and MPO on neutrophil exposed to AECs grown on inserts with a $0.4 \mu \mathrm{m}$ pore size did not increase and, in fact, was no different to the values obtained in the absence of AECs.

Neutrophils recovered from the basolateral side of the RSV infected AEC cultures grown on $0.4 \mu \mathrm{m}$ inserts demonstrated CD11b, CD64, CD62L, NE and MPO levels that were at least $30 x$ lower compared to those incubated on AECs grown on inserts with $3 \mu \mathrm{m}$ pores (Figure 4). This reduction in neutrophil activation was significant $(p<0.05)$ across all groups tested (mock and RSV infected) suggesting the increased expression of these markers is a result of migration rather than infection. 


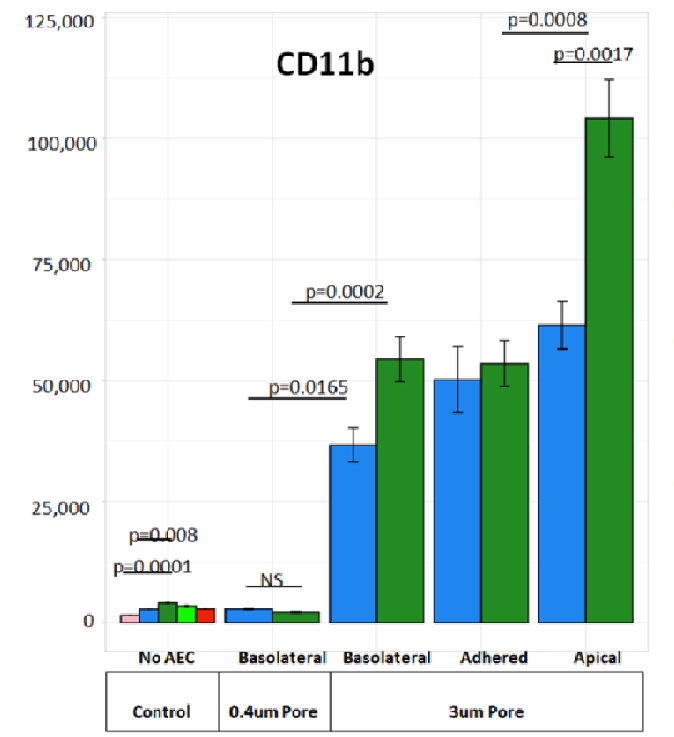

D

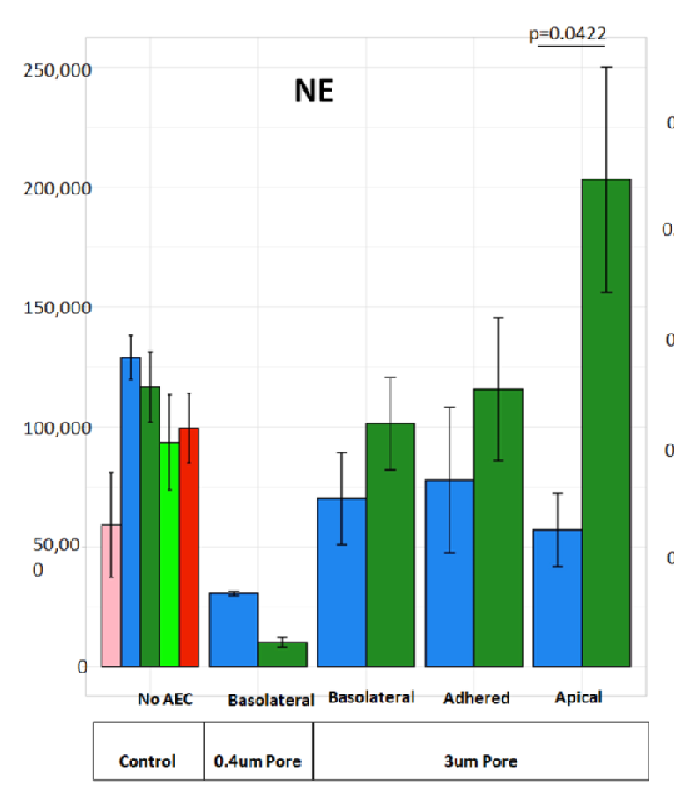

$0.3 \mathrm{M}$

$0.2 \mathrm{M}$

$0.1 \mathrm{M}$

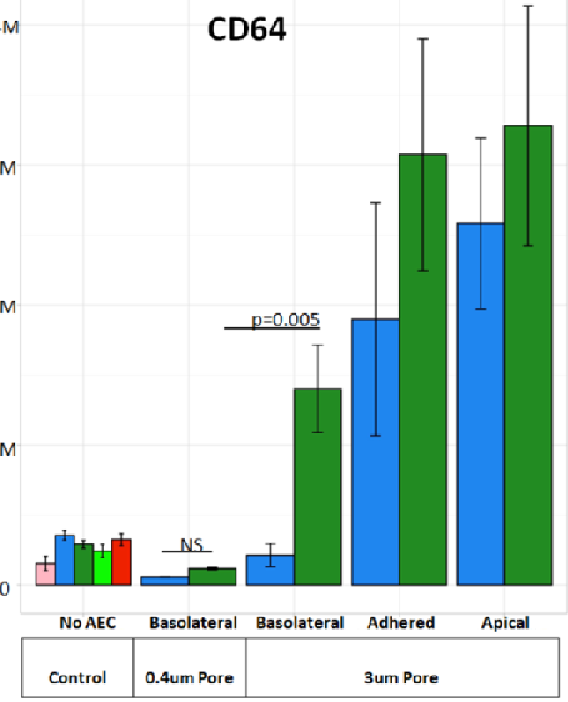

E

CD64

C

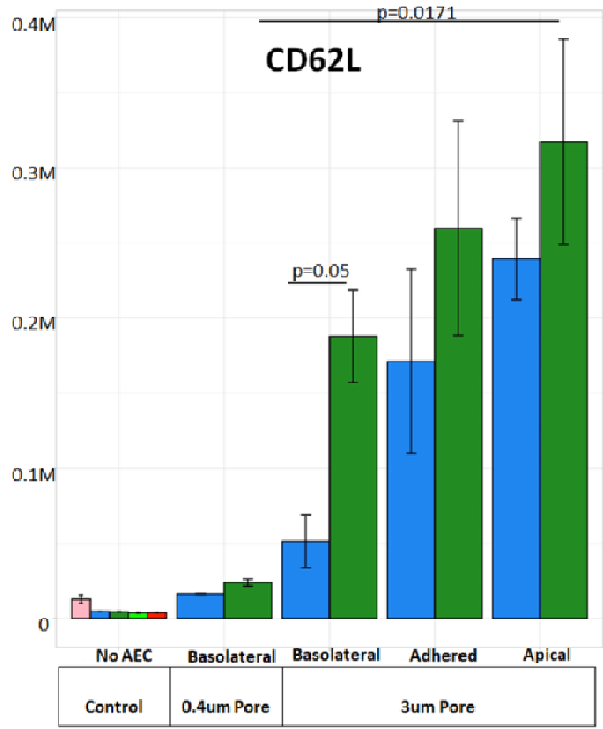

0

MPO

$0.4 \mathrm{M}$

$0.3 \mathrm{M}$

$0.2 \mathrm{M}$

$0.1 \mathrm{M}$

0

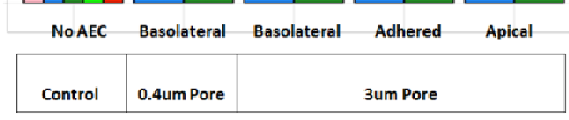

4 Neutrophils undergoin trans-epithelial migration alter $t$ expression of surface markers

depending on their spatial locat

(A-E) Mean fluorescence intensity cell surface expressed CD11b (A) CD64 (B), CD62L (C), NE (D), MP

(E) on neutrophils incubated with media alone (No AEC) or $1 \mathrm{~h}$ follov migration across mock 'infected' A or RSV infected AEC cultures infe for 72 hours on membrane inserts a $3 \mu \mathrm{m}$ pore size or those grown o membrane inserts with $0.4 \mu \mathrm{m}$ por which prevents cellular migration Supplementary Figure S2). Grar are separated to distinguish neutrophils recovered from the basolateral, adherent, and apical (migrated) compartments within th assay. Neutrophils incubated with $f$ and RSV MOI 1 (virus) in media without AEC were also used as controls. Bars indicate standard e of the mean. A linear model of mix effects was used to compare interactions between infection and ocation groups and control for int donor variability. Individual comparisons performed with TwoANOVA with pairing and Tukey's hock test. ${ }^{*}=$ in comparison to respective basolateral group \# = ir comparison to respective adheren group. 


\section{Discussion}

This study has, for the first time, observed and measured neutrophil movement, spatially and temporally, during migration across human AECs in response to RSV infection. We found that RSV infection led to greater, but slower, net movement of neutrophils across RSV infected AECs in comparison to mock infected AECs, with greater adherence of neutrophils to the RSV infected AECs occurring during the first 15-18 minutes, in small aggregates or clusters. Secretion of soluble granular factors including MMP9, MPO, and neutrophil elastase, were greater in the apical than the basolateral compartments, under all conditions of migration, which suggested that migration per se was associated with increased release of these factors. We found that the expression of CD11b, CD62L, CD64, NE and MPO, on the neutrophils, was increased in all compartments of our system when migration occurred and this was greater, for NE and MPO expression, on neutrophils in the apical compartment when AECs had been infected with RSV. We also found evidence suggesting that migrated, activated neutrophils can reverse migrate to the basolateral membrane.

In RSV bronchiolitis, neutrophil activation has been shown as a key precursor to the development of severe respiratory symptoms ${ }^{25}$. The primary site of attachment of RSV is the $A E C$, the cell in which the virus establishes replicative infection ${ }^{26}$. Viraemia due to RSV has not, to our knowledge, been observed clinically, which suggests that the virus causes symptoms because of its effects on the respiratory tract. Neutrophils are recruited from the systemic circulation to the respiratory tract in response to RSV infection. Clinical studies of children hospitalised with RSV bronchiolitis have shown neutrophils with upregulated CD11b were recoverable both from the airways in BAL and from peripheral circulation? ${ }^{9}$.

Furthermore, neutrophils recovered from peripheral circulation of infants with RSV bronchiolitis have been shown to contain RSV mRNA ${ }^{11}$. This poses important questions, which this study has attempted to address. Firstly, are activated neutrophils preferentially selected for migration or does the process of trans-epithelial migration increase expression of neutrophils activation markers? And secondly, how do RSV transcripts become present in circulating neutrophils?

In this study we explored the interaction between neutrophils and RSV infected AECs in more detail using a novel in vitro model. Here, we found that RSV infection led to a greater net movement of neutrophils across RSV infected AECs in comparison to mock infected AECs. We found that RSV infection led to greater numbers of neutrophils remaining adherent to RSV infected AECs. This may be due to increased expression of host cell 
receptors including ICAM-1 (intercellular adhesion molecule 1), which we (and others) have previously shown to be upregulated in vitro airway models of RSV infection, and mediate neutrophil adherence to RSV infected AECs ${ }^{22} 27$. In support of this, we found that fewer neutrophils remained adherent to mock infected AECs exposed to RSV infected supernatant placed apically (in comparison to RSV infected AECs). This suggests that properties of the epithelial cells, rather than the apical milieu of the RSV infected epithelium per se, are responsible for neutrophil adherence. Interestingly, neutrophils were observed to adhere in clusters to the apical surface of RSV infected AECs. Neutrophil clustering has been shown as a vital mechanism for host defence against pathogens ${ }^{22,28}$. However, large clusters have been associated with inflammatory disease in both human and murine models ${ }^{29,30}$. In models of RSV infection, increased neutrophil adherence and clustering has been associated with greater AEC damage ${ }^{21,22,31,32}$. To examine the formation of these clusters in more detail, we used fast time-lapse microscopy to image the early movement of neutrophils moving across RSV infected AECs. This showed that clusters can result from the coordinated convergence of neutrophils, which resembled the neutrophil swarming reported previously in in vivo models notably in mice and zebrafish in response to infection and sterile injury ${ }^{23,24,22}$. This pattern of neutrophil movement is thought to be initiated by the release of danger-associated molecular patterns (DAMPs) from neutrophils, which induce a transcriptional switch in their neighbours and coordinate a collective movement ${ }^{33}$. LTB4 is a leukotriene DAMP, released by dying neutrophils and has been shown to mediate this directed leukocyte movement previously $24,29,34,35$. Here, we speculate that the formation of clusters of neutrophils, seen in our model, is not mediated by the RSV infected AECs, but instead due to neutrophils themselves triggering and maintaining clustering behaviour. The ability of neutrophils to affect others around them is an emerging area of research, and neutrophil quorum signalling has been shown to coordinate neutrophil collective responses to wound healing ${ }^{23,24,36}$. Whether this response is a natural protective mechanism in countering an RSV infection or an aberrant response remains to be fully explained, but focal damage to AECs in vivo may allow increased neutrophil migration.

By tracking the movement of individual neutrophils in 3D, we found that neutrophils migrated across RSV infected AECs more slowly and covered less distance compared to those migrating across mock infected AECs. However, if neutrophils were exposed to AEC supernatants alone, in the absence of AECs, then we found that the neutrophils moved faster towards RSV infected supernatants, compared to neutrophils exposed to mock infected AEC supernatants. This is consistent with our finding that the apical supernatant of RSV infected AECs contain elevated levels of CXCL8 (IL-8) and CXCL10 (IP-10), both potent neutrophil chemoattractants, compared to the mock infected AECs (Supplementary 
Figure S3). Therefore, we hypothesise that an airway epithelial cell factor is responsible for slowing down the migration of neutrophils in the RSV infected AEC model. This may be mediated by the interaction of neutrophil integrins such as LFA-1 and upregulated ICAM-1, as we have shown before in a similar model ${ }^{27}$. An alternative suggestion is that a known histopathological characteristic of RSV infection, cell syncytia formation, could reduce cellcell junctions through which migration may occur ${ }^{37}$. The formation of syncytia and reduced availability of accessible tight junctions may contribute to increased impedance of RSV infection AECs to neutrophil transepithelial migration in comparison to mock infected $A E C s^{27,37}$. This may not only slow down the initial neutrophil response to RSV infection, but also prolong its duration and slow its resolution, contributing to a heightened period of inflammation.

To address the question of whether trans-epithelial migration increases expression of neutrophils activation markers, such as those seen in BAL recovered from infants with RSV bronchiolitis, we measured the surface expression of CD11b, CD64, CD62L, NE and MPO on neutrophils from all compartments of our model. Here we found that CD11b, CD64, CD62L, NE and MPO were upregulated as a direct consequence of trans-epithelial migration across AECs, with highest expression of all markers recorded on neutrophils recovered from the apical compartment of RSV infected AECs. Interestingly, neutrophils recovered from basolateral compartments of the AEC model also increased expression of CD11b, CD64, CD62L and MPO, but not NE, compared to the no-AEC control, which at first indicated to us that possible secreted factors from basolateral side of AECs may increase cell marker activation. However, when we cultured our AEC model using membrane inserts with a small pore size $(0.4 \mu \mathrm{m})$, that prevented neutrophil migration, we did not find any difference in CD11b, CD64, CD62L and MPO expression on basolateral neutrophils compared to neutrophils exposed to media alone (no AECs). This suggests that neutrophils increase their expression of CD11b, CD64, CD62L and MPO during or following migration, rather than an existing sub-population of activated neutrophils, already expressing greater levels of these markers, being selected for migration. This was different for NE. Here we detected a decrease in NE expression when neutrophils were exposed to basolateral side of RSV infected AECs, compared to mock infected. as a similar pattern in NE expression is seen when comparing neutrophils exposed to the no-AEC mock and RSV supernatants, suggesting that secreted factors that drive this change.

Interestingly, the presence of these 'activated' neutrophils on the basolateral side of the AECs after $1 \mathrm{~h}$, suggests that neutrophil migration through AECs may be bidirectional, and that migrated neutrophils, which are 'activated' by the apical environment, reverse migrate to 
return to the basolateral side of AECs (see Graphical Abstract). To support this theory, we performed some preliminary analysis the Z-tracks of neutrophils migrating across RSV infected AECs (Supplementary Figure S4) using spinning disk confocal microscopy. This showed that neutrophils can move bidirectionally across the AECs, quickly migrating to the apical side of RSV infected airway epithelium and returning to the basolateral compartment, as soon as 15 minutes after migration.

In summary we have shown that neutrophils show distinct, measurable patterns of movement during trans-epithelial migration through RSV infected AECs, including the formation of neutrophil clusters and upregulation of CD11b expression. We present evidence of bidirectional movement of neutrophils during RSV infection that could have important systemic implications for severe disease sequalae, and indeed go some way to explain the increased expression of CD11b and other markers and presence of viral products in blood neutrophils of babies with RSV bronchiolitis found in previous clinical studies ${ }^{38} 9$ 11, 23,33,36. This is a critical area for discovery and the model that we have developed here could be used to unravel important disease mechanisms, including the key question of how RSV accesses extra-pulmonary sites during infection. For example, it could be used to identify new biomarkers of specific neutrophils sub-populations responsible for driving severe disease outcomes; to screen anti-inflammatory drugs; or determine new mechanisms that aid disease resolution. 


\section{Materials and Methods}

A step-by-step protocol of this methodology is deposited in Protocol Exchange.

\section{Participants}

Peripheral blood and airway epithelial cells were obtained from healthy adult donors at UCL GOS Institute of Child Health. Written informed consent was obtained from all donors prior to their enrolment in the study. Study approval was obtained from the UCL Research Ethics Committee (4735/002). All methods were performed in accordance with the relevant guidelines and regulations.

\section{Neutrophil Isolation}

Venous blood was collected in EDTA (Ethylene Diamine Tetra Acetic) tubes (Greiner). Neutrophils were then ultra-purified using an EasySep Direct Neutrophil isolation kit (Stem Cell Technologies) according to the manufacturer's instructions and subsequently stained with Cell Trace Calcein Red-Orange cell stain (ThermoFisher) and processed as described previously ${ }^{39}$.

\section{Transepithelial Migration Model}

This study modified the transepithelial migration model described by Herbert et al (2020) to utilise undifferentiated human AECs, grown at air liquid interface for 7 days as opposed to 28-day ciliated cultures. This ensures a flatter more uniform culture which is possible to live image using confocal microscopy ${ }^{22}$. AECs cells were cultured on porous PET inserts (Greiner) with pore size of $3 \mu \mathrm{m}$ to allow migration or $0.4 \mu \mathrm{m}$ to prohibit it. AEC cultures were infected with RSV 24 or 72 hours prior to the addition of neutrophils. Mock infected AECs with RSV infected AEC supernatant were used as a control (RSV Sup), as were mock infected AECs with n-Formylmethionine-leucyl-phenylalanine (fMLP) 100nM placed apically as a positive control for neutrophil chemotaxis. $400 \mu$ of supernatant was added underneath the membrane insert for each experimental group, Mock, RSV, RSV supernatants only and fMLP control. Excess supernatant prior to neutrophil migration was stored at -20 for use in chemotaxis and activation experiments. Neutrophils were then added to the basolateral side of all membrane inserts, then left to incubate for 1 or 4 hours. After migration, neutrophils were collected from the apical side of the epithelial cells for quantification. Supernatants 
were collected and membrane inserts were fixed and stained. Mock infection was performed by inoculating with sterile media in the place of viral inoculum.

\section{Virus purification and quantification}

Recombinant GFP tagged RSV A2 strain was kindly provided by Jean-Francois Eleouet and described in Fix et $\mathrm{al}^{40}$. Viral stock preparation and quantification of viral titre was performed using HEp-2 cells (ATCC CCL-23) was performed as described previously ${ }^{22}$.

\section{Microscopy}

AEC cultures were fixed for microscopy using 4\% PFA (v/v) and mounted using Propyll Gallate. Images of fixed cultures were acquired using an inverted Zeiss LSM 710 confocal microscope using a 20x Plan Achromat LWD objective. Live cell imaging was performed using a Perkin Elmer UltraView spinning disk (CSU22) confocal microscope. All microscopes were located in the UCL GOS Institute of Child Health Imaging Facility (London, UK).

\section{Quantification of migrated and adherent neutrophils}

The number of migrated neutrophils was quantified as described previously ${ }^{22}$. Flow cytometric analysis of CD11b, NE, MPO, CD64 and CD62L expression was performed as described previously ${ }^{21}$. Antibodies used are provided in supplementary information. Image acquisition was the same as above. Neutrophils were counted using an ImageJ counting tool.

\section{Quantifying neutrophil distribution}

To investigate whether neutrophil adherence to AECs was uniform (null hypothesis $=0$ ) or whether they clustered, a method of measuring neutrophil distribution was required. Images taken of AECs after 1 hour of migration were taken as described previously, then analysed using ImageJ to first identify neutrophils within the image, determine their 3D coordinates. These coordinates were then exported into RStudio and analysed using a spatial statistics package to then calculate the distance between the centre-point of each neutrophil and the centre-point of its five nearest neighbours ${ }^{41}$. However, it is expected that if more neutrophils are adherent to RSV infected AECs in comparison to mock, that they would be closer together by crowding alone. To account for this, the distance apart each neutrophil would be if neutrophils were evenly spread over the AEC area was calculated from the numbers of neutrophils counted as adherent to the same AECs. ImageJ macro code for determining 3D coordinates and subsequent $\mathrm{R}$ script for distance analysis is available upon request.

\section{Analysis of neutrophil chemotaxis}


Time-lapse videos were analysed using Icy, programmed to run a spot detection protocol for each frame of the stack, detecting light objects close to $10 \mu \mathrm{m}$ diameter on a dark background to identify neutrophils within the image. Then, a spot tracking plugin was used to map the displacement of each neutrophil through each time point image ${ }^{42}$. The tracks produced were then visually checked for tracking accuracy and where erroneous tracks were found, corrected manually, or removed from analysis. Raw spot files and track files were exported for further analysis using RStudio. Data sets detailing total displacement, net displacement, average, maximum and minimum speed, and linearity were exported combined with replicate data sets for analysis using RStudio and GraphPad Prism v8.0.

Time-lapse imaging of neutrophil trans-epithelial migration in $3 D$

A black plastic 24 well plate with a glass coverslip bottom (Greiner) was used in place of a low binding 24 well plate (Greiner) to allow for live imaging of AEC cultures during transepithelial migration. Neutrophils were identified using Icy and a spot detection protocol initiated for each frame of the stack, detecting light objects close to $10 \mu \mathrm{m}$ diameter on a dark background. Then each spot was tracked using the spot tracking plugin using a 3D tracking algorithm ${ }^{42}$. Tracks were then visually checked for tracking accuracy and where erroneous tracks were found, were either corrected manually or removed from analysis. Raw track coordinates were exported for further analysis using RStudio package Motility Lab ${ }^{43}$. Data detailing displacement, speed and linearity were exported. Analysis of neutrophil movement and distribution was performed using RStudio packages 'motilitylab', 'spatstat' and 'dyplr'. 


\section{References}

1. Glezen, W. P., Taber, L. H., Frank, A. L. \& Kasel, J. A. Risk of primary infection and reinfection with respiratory syncytial virus. Am. J. Dis. Child. 140, 543-6 (1986).

2. Hall, C. B. et al. The Burden of Respiratory Syncytial Virus Infection in Young Children. N. Engl. J. Med. 360, 588-598 (2009).

3. Smyth, R. L. \& Openshaw, P. J. Bronchiolitis. Lancet vol. 368 312-322 (2006).

4. Shi, T. et al. Global, regional, and national disease burden estimates of acute lower respiratory infections due to respiratory syncytial virus in young children in 2015: a systematic review and modelling study. Lancet 390, 946-958 (2017).

5. Nair, H. et al. Global burden of acute lower respiratory infections due to respiratory syncytial virus in young children: a systematic review and meta-analysis. Lancet $\mathbf{3 7 5}$, 1545-1555 (2010).

6. Geoghegan, S. et al. Mortality due to respiratory syncytial virus. burden and risk factors. Am J Respir Crit Care Med 195, 96-103 (2017).

7. McNamara, P. S., Ritson, P., Selby, A., Hart, C. A. \& Smyth, R. L. Bronchoalveolar lavage cellularity in infants with severe respiratory syncytial virus bronchiolitis. Arch. Dis. Child. 88, 922-926 (2003).

8. Openshaw, P. J. \& Chiu, C. Protective and dysregulated T cell immunity in RSV infection. Current Opinion in Virology vol. 3 468-474 (2013).

9. Halfhide, C. P. et al. Neutrophil TLR4 expression is reduced in the airways of infants with severe bronchiolitis. Thorax 64, 798-805 (2009).

10. Openshaw, P. J. M. M., Chiu, C., Culley, F. J. \& Johansson, C. Protective and Harmful Immunity to RSV infection. Annu. Rev. Immunol. 35, 501-532 (2017).

11. Halfhide, C. P. et al. Respiratory syncytial virus binds and undergoes transcription in neutrophils from the blood and airways of infants with severe bronchiolitis. $J$ Infect Dis 204, 451-458 (2011).

12. Smyth, R. L., Mobbs, K. J., O’Hea, U., Ashby, D. \& Hart, C. A. Respiratory syncytial virus bronchiolitis: Disease severity, interleukin-8, and virus genotype. Pediatr. Pulmonol. 33, 339-346 (2002).

13. Bataki, E. L., Evans, G. S. \& Everard, M. L. Respiratory syncytial virus and neutrophil activation. Clin Exp Immunol 140, 470-477 (2005).

14. Erhlich, P. \& Lazarus, E. Die Anaemie. Wein 8, (1898).

15. Chin, A. C. \& Parkos, C. A. Pathobiology of neutrophil transepithelial migration: implications in mediating epithelial injury. Annu Rev Pathol 2, 111-143 (2007).

16. Lyck, R. \& Enzmann, G. The physiological roles of ICAM-1 and ICAM-2 in neutrophil migration into tissues. Curr. Opin. Hematol. 22, 53-59 (2015).

17. REBUCK, N., GIBSON, A. \& FINN, A. Neutrophil adhesion molecules in term and premature infants: normal or enhanced leucocyte integrins but defective L-selectin expression and shedding. Clin. Exp. Immunol. 101, 183-189 (2008).

18. Papayannopoulos, V. Neutrophil extracellular traps in immunity and disease. Nat. Rev. Immunol. 18, 134-148 (2018).

19. Cannon, M. J., Openshaw, P. J. M. \& Askonas, B. A. Cytotoxic T cells clear virus but augment lung pathology in mice infected with respiratory syncytial virus. J. Exp. Med. 168, 1163-1168 (1988).

20. Elawady, S. et al. Neutrophil CD64 as a diagnostic marker of sepsis in neonates. $J$. Investig. Med. 62, 644-649 (2014).

21. Deng, Y. et al. Neutrophil: Airway Epithelial Interactions Result in Increased Epithelial Damage and Viral Clearance during RSV Infection. J. Virol. (2020) doi:10.1128/jvi.02161-19.

22. Herbert, J. A. et al. $\beta 2$ integrin LFA1 mediates airway damage following neutrophil trans-epithelial migration during RSV infection. Eur. Respir. J. 1902216 (2020) doi:10.1183/13993003.02216-2019.

23. Poplimont, H. et al. Neutrophil Swarming in Damaged Tissue Is Orchestrated by Connexins and Cooperative Calcium Alarm Signals. Curr. Biol. (2020) doi:10.1016/j.cub.2020.05.030. 
24. Lämmermann, T. et al. Neutrophil swarms require LTB4 and integrins at sites of cell death in vivo. Nature 498, 371-375 (2013).

25. MS, H. et al. Neutrophilic inflammation in the respiratory mucosa predisposes to RSV infection. Science 370, (2020).

26. Zhang, L., Peeples, M. E., Boucher, R. C., Collins, P. L. \& Pickles, R. J. Respiratory Syncytial Virus Infection of Human Airway Epithelial Cells Is Polarized, Specific to Ciliated Cells, and without Obvious Cytopathology. J. Virol. 76, 5654-5666 (2002).

27. Wang, S. Z. et al. Adhesion molecule expression on epithelial cells infected with respiratory syncytial virus. Eur. Respir. J. 15, 358-366 (2000).

28. Yonker, L. M. et al. Development of a Primary Human Co-Culture Model of Inflamed Airway Mucosa. Sci Rep 7, 8182 (2017).

29. Song, Z. et al. NADPH oxidase controls pulmonary neutrophil infiltration in the response to fungal cell walls by limiting LTB4. Blood 135, 891-903 (2020).

30. Alex, $\mathrm{H}$. et al. Neutrophil swarming delays the growth of clusters of pathogenic fungi. Nat. Commun. 11, (2020).

31. Grommes, J. \& Soehnlein, O. Contribution of neutrophils to acute lung injury. Mol. Med. 17, 293-307 (2011).

32. Wang, S. Z. et al. Neutrophils induce damage to respiratory epithelial cells infected with respiratory syncytial virus. Eur. Respir. J. 12, 612-8 (1998).

33. Vanhook, A. M. Coordinating a neutrophil swarm. Science Signaling vol. 13 (2020).

34. Subramanian, B. C., Majumdar, R. \& Parent, C. A. The role of the LTB4-BLT1 axis in chemotactic gradient sensing and directed leukocyte migration. Seminars in Immunology vol. 33 16-29 (2017).

35. Wan, M., Tang, X., Stsiapanava, A. \& Haeggström, J. Z. Biosynthesis of leukotriene B4. Seminars in Immunology vol. 33 3-15 (2017).

36. Palomino-Segura, M. \& Hidalgo, A. Immunity: Neutrophil quorum at the wound. Curr. Biol. 30, R828-R830.

37. Tian, J. et al. RAGE inhibits human respiratory syncytial virus syncytium formation by interfering with F-protein function. J. Gen. Virol. 94, 1691-700 (2013).

38. Halfhide, C. P. et al. Respiratory Syncytial Virus Binds and Undergoes Transcription in Neutrophils From the Blood and Airways of Infants With Severe Bronchiolitis. J. Infect. Dis. 204, 451-458 (2011).

39. Deng, Y., Herbert, J. A., Smith, C. M. \& Smyth, R. L. An in vitro transepithelial migration assay to evaluate the role of neutrophils in Respiratory Syncytial Virus (RSV) induced epithelial damage. Sci. Rep. 8, 6777 (2018).

40. Fix, J. The Insertion of Fluorescent Proteins in a Variable Region of Respiratory Syncytial Virus L Polymerase Results in Fluorescent and Functional Enzymes But with Reduced Activities. Open Virol. J. 5, 103-108 (2011).

41. Bolte, S. \& Cordelières, F. P. A guided tour into subcellular colocalization analysis in light microscopy. Journal of Microscopy vol. 224 213-232 (2006).

42. Chenouard, N., Bloch, I. \& Olivo-Marin, J. C. Multiple hypothesis tracking for cluttered biological image sequences. IEEE Trans. Pattern Anal. Mach. Intell. 35, 2736-2750 (2013).

43. Dannenberg, K., Berry, J., Maintainer, J. T. \& Textor, J. Package 'MotilityLab' Type Package Title Quantitative Analysis of Motion. (2016) doi:10.1038/nri2638. 


\section{Acknowledgements}

LR was recipient of a Newton fellowship from The Academy of Medical Science (ref NIF004/1012). RLS was supported by the Great Ormond Street Children's Charity (grant code W1802). CMS is currently a recipient of grants from Animal Free Research UK (AFR19-20274), BBSRC (BB/V006738/1), GOSH Children's charity (COVID_CSmith_017) and the Wellcome Trust (212516/Z/18/Z). This research was supported by the NIHR Great Ormond Street Hospital Biomedical Research Centre. Microscopy was performed at the Light Microscopy Core Facility, UCL GOS Institute of Child Health supported by the NIHR GOSH BRC award 17DD08. The views expressed are those of the author(s) and not necessarily those of the NHS, the NIHR or the Department of Health. We thank Dr Shyam Sawhney for technical support and the healthy volunteers who donated airway cells and blood samples for this study

\section{Author contributions}

All authors declare no conflicts of interest.

ER conceived and designed the study, conducted experiments, analysed data, and prepared the manuscript. JAH designed the study, conducted experiments, analysed data, and reviewed the manuscript. MP assisted with flow cytometry data analysis and review of the manuscript. LR, IL, and AP assisted with data collection and review of the manuscript. DM assisted with microscopy acquisition and reviewed the manuscript. MC-B conducted statistical analysis and reviewed the manuscript. RLS and CMS oversaw the funding application and contributed to study conception and design, data analysis and interpretation, and the write-up of the manuscript. 
Inserts with a $3 \mu \mathrm{m}$ pore size permit trans-epithelial migration

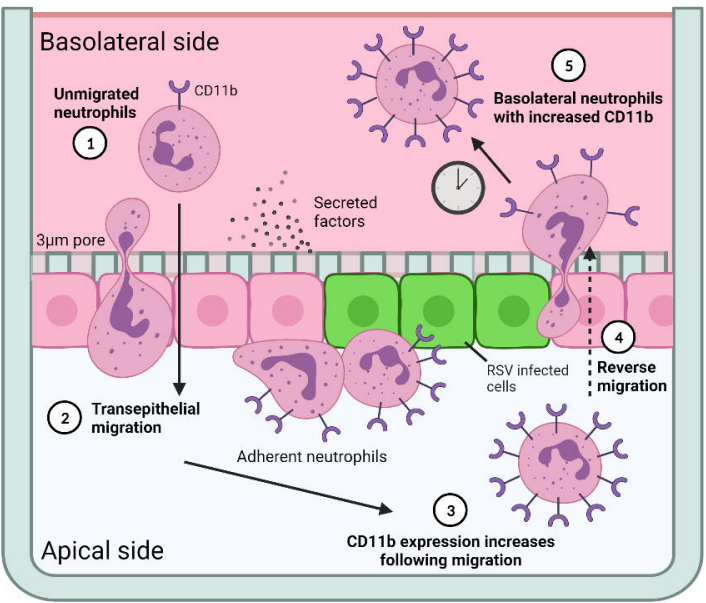

\section{$0.4 \mu \mathrm{m}$ pores prevent trans-epithelial migration}

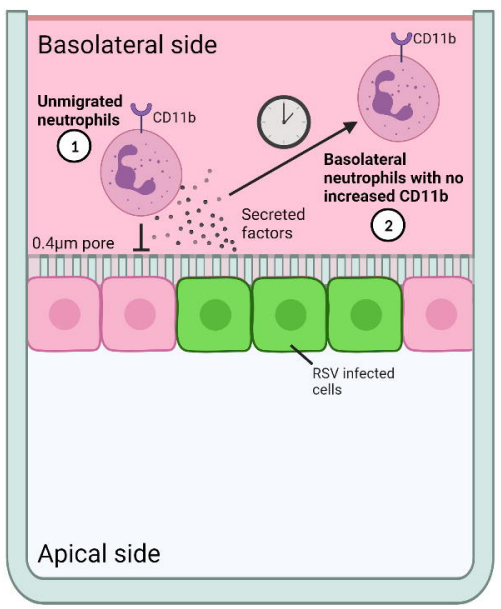


air-liquid interface

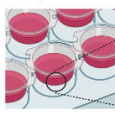

3. Add RFPeneutrophils

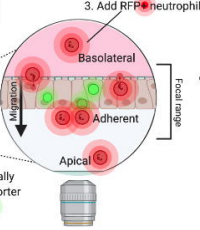

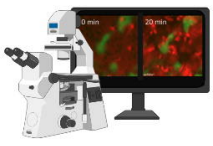

2. Infect apically with GFP+ reporter

RSV 

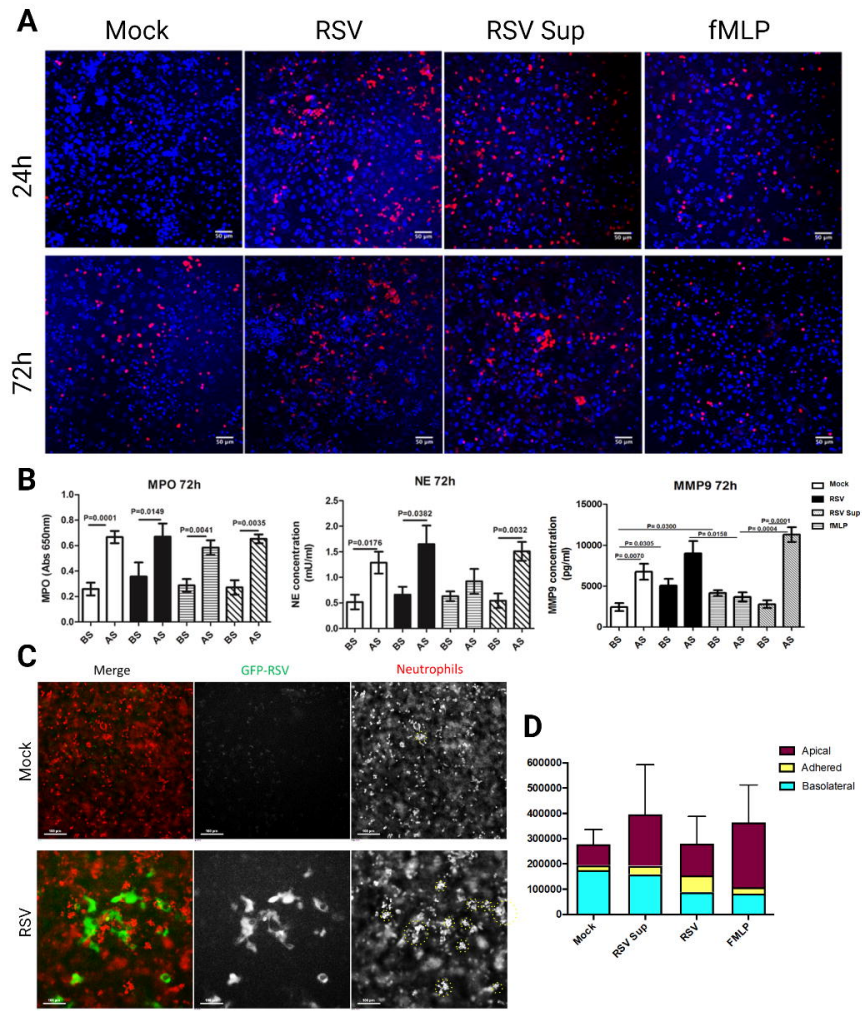

E

F
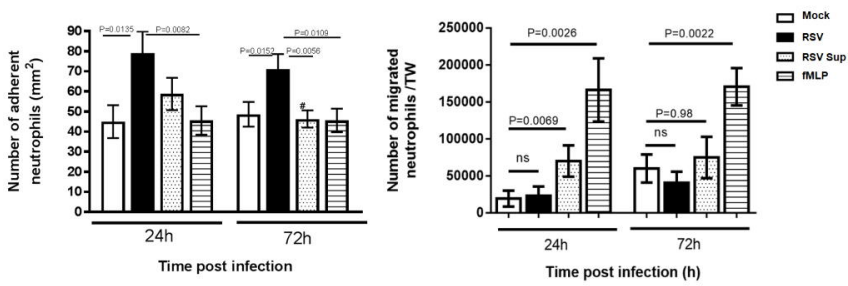
A
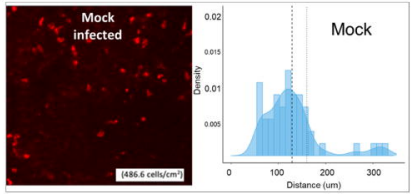

c

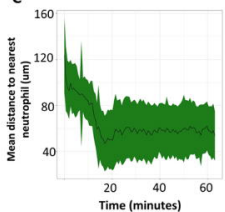

F

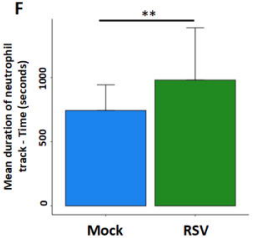

B
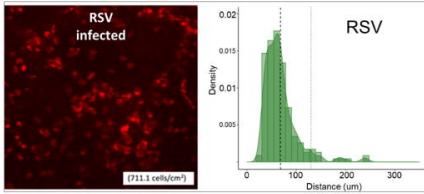

E
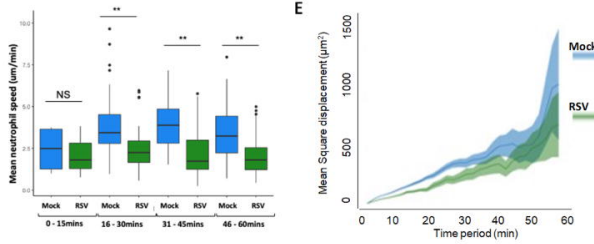

G

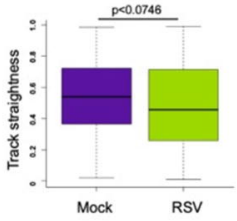


